\title{
EBSD Spatial Resolution in the SEM when Analyzing Small Grains or Deformed Material
}

\author{
P. Rolland*, K Dicks*, R. Ravel-Chapuis** \\ *Oxford Instruments Analytical, Halifax road, High Wycombe HP12 3SE UK \\ **Jeol Europe SA rue E. Labiche, Allée de Giverny 78290Croissy sur Seine France
}

The maximum spatial resolution that can be achieved by Electron BackScattered Diffraction (EBSD) in the Scanning Electron Microscope (SEM) is highly dependant on the electron probe diameter, correction for aberrations and the current density at the specimen surface. These parameters dictate the pattern quality of the Electron Backscatter Pattern (EBSP). In practice this means using the minimum beam current, i.e smallest spot size, consistent with acquiring solvable patterns. This limit is dependant on SEM performance and EBSD sensitivity, i.e. the more sensitive the EBSD camera, the lower the beam current that can be successfully used. Further, the grain size and condition of the grain boundaries of the sample has an effect. The minimum grain size that can be resolved depends on the boundary condition - If the grain boundary is strain free, a resolution of $10 \mathrm{~nm}$ may be achieved. However, strain present at the boundary may degrade resolution by a further 20 to $70 \mathrm{~nm}$ beyond that theoretically achievable. Deformed materials typically have highly strained boundaries. In this study undeformed and highly deformed intestitial free steel was examined.

F. J. Humphreys (1) defined an "effective spatial resolution" as the distance over which an Electron Backscattered Pattern (EBSP) cannot be indexed when crossing a boundary. This assumes that the resolution is limited by the simultaneous acquisition of two EBSPs, which consequently cannot be indexed. Spatial resolution is highly dependant on the boundary type. See figure 1. A strain free boundary such as a CSL sigma 3 shows every pixel solved even when steps as small as $5 \mathrm{~nm}$ are used on a Tungsten gun SEM. Conversely, under similar conditions, poorer resolution occurs when a strained boundary is crossed. When approaching such a boundary, EBSP quality degrades until no pattern occurs at the boundary. On passing into the second grain, EBSP quality improves again until pattern indexing is restored.

However, close inspection shows that when crossing a strain free boundary, the orientation results from grains either side of the boundary become substituted. This results from the fusion of patterns from both the grains, i.e. the electron beam straddles the boundary. This is the real "effective resolution". In both strained or unstrained grain boundaries, the width of the region where information is mixed or lacking is dependant on the beam current as seen in figure 2 .

In both cases the 'quality of focusing' (i.e. spot size, control of aberrations etc.) is critical to form 'clean', unmixed patterns. It is crucial to avoid degrading a good EBSP with a blank or poor EBSP at the strained boundary or conversely, mix 2 or more EBSP's of changing orientation. Figure 3 shows data acquired on a W-SEM with grains as small as $100 \mathrm{~nm}$. Figure 4 shows results obtained on a sample deformed by laser beam. Only the FEG SEM allowed resolution of the fine orientation changes, pixel by pixel. Using either SEM, increasing beam current eventually lead to poor/no EBSP. 


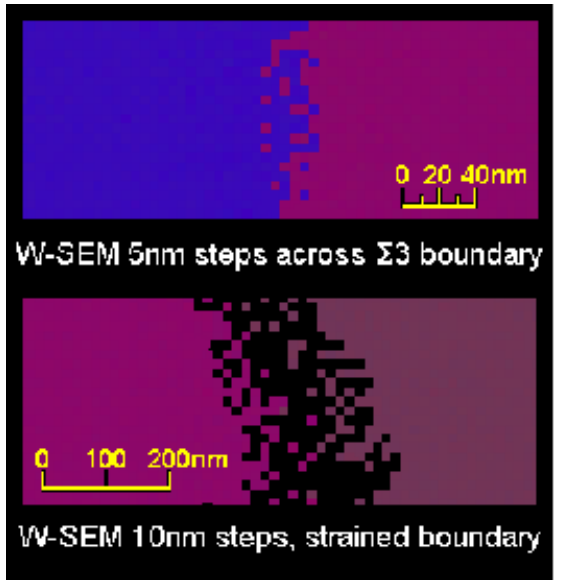

Figure 1

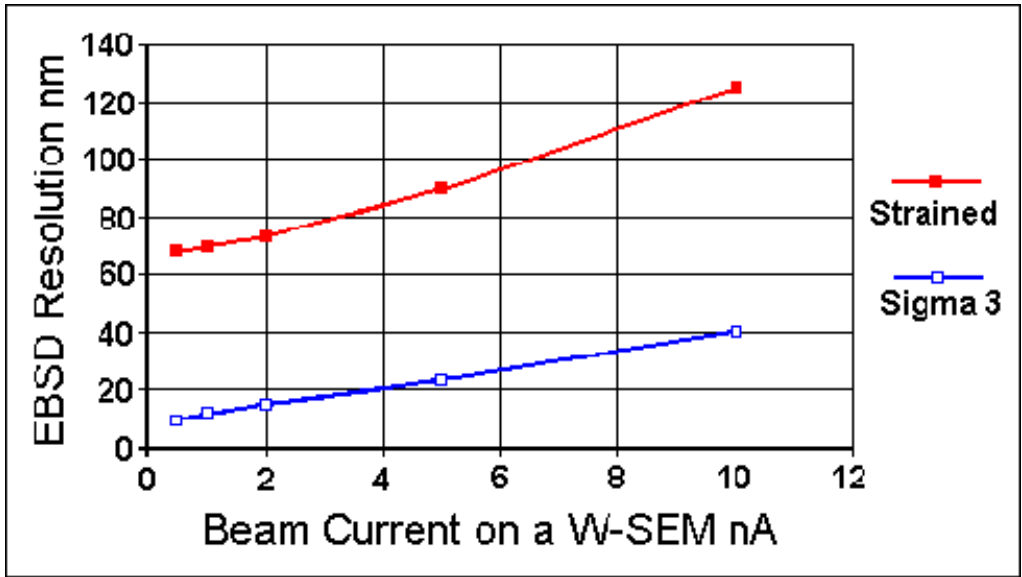

Figure 2

Figure 1, top: COM of a CSL $\Sigma 3$ boundary, undeformed steel, acquired at 350pA beam current. All EBSPs have solved. A $\Sigma 3$ boundary is expected to be straight, strain free and therefore clearly delineated. However, some substitution of pixels has occurred between the left and right grains at the $\Sigma 3$ boundary. The electron probe diameter is such that EBSP's from either side of the boundary have become superimposed, leading to ambiguous solutions.

Figure 1, bottom: shows a COM for a nearby boundary ( $43^{\circ}$ misorientation) in the same undeformed steel, acquired at 350pA. Note that the EBSP's have not solved at the boundary, indicative of strain. However, there is no substitution of solutions either side of the boundary, unlike the case of the $\Sigma 3$ above, where the probe diameter was the limiting factor. At this strained boundary, the progressive degradation of EBSP quality due to strain causes failure to solve - with the apparent difference of EBSD resolution, even though the electron optical conditions were unchanged.

Figure 2 EBSD resolution on a W-SEM plotted vs. beam current (nA), (30ms camera integration time) for strained and unstrained boundaries. Note the loss of resolution as the beam current is increased. The minimum beam current required to solve EBSP's was 350 pA.
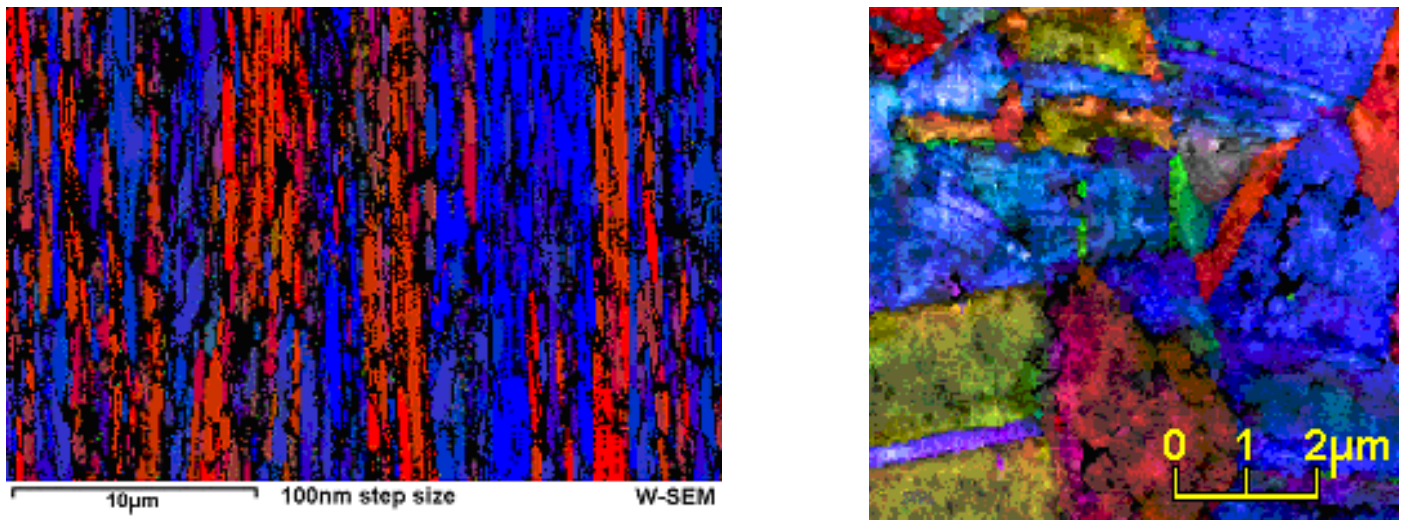

Figure 3: COM acquired using a W-SEM. Note loss of information at grain boundaries where strain is greatest and EBSP quality is degraded
Figure 4: COM acquired using a FEG-SEM on a deformed steel, $50 \mathrm{~nm}$ steps. The typical misorientation between adjacent pixels at this resolution is $<3^{\circ}$ i.e. the continuously changing orientation information is preserved. Using W-SEM \& $50 \mathrm{~nm}$ step size the hit rate decreases to $40 \%$ and the continuity of information is lost

1 F.J. Humphreys and J. Brough Microscopy Society of America 1999 Proceedings pages 240-241 